\title{
Accurate inter-vehicle distance measurement based on monocular camera and line laser
}

\author{
Seungnam Kang ${ }^{\mathrm{a})}$, Insub Yoo, Myungok Shin, and Seungwoo Seo ${ }^{\mathrm{b})}$ \\ Department of Electrical and Computer Engineering, Seoul National University, \\ Seoul 151-742, Korea \\ a) snkang@cnslab.snu.ac.kr \\ b) sseo@snu.ac.kr
}

\begin{abstract}
Inter-vehicle distance measuring technique is widely used for Driving Assistance System (DAS) such as Adaptive Cruise Control (ACC) and Forward Collision Warning (FCW) which are useful for safety enhancement as well as driving convenience. In this paper, we propose a new method for providing accurate longitudinal inter-vehicle distance measurement by using a monocular camera and a line laser. Experimental results show that the proposed method outperforms the disparity-based method and the error in distance measurement is low enough even in nighttime as much as daytime.
\end{abstract}

Keywords: inter-vehicle distance measurement, monocular, line laser Classification: Electronic instrumentation and control

\section{References}

[1] D. O. Cualain, M. Glavin, E. Jones and P. Denny: Irish Signals and Systems Conference (2007).

[2] D. Gallup, J. M. Frahm, P. Mordohai and M. Pollefeys: Computer Vision and Pattern Recognition (2008) 1. DOI:10.1109/CVPR.2008.4587671

[3] N. Jojic, B. Brumitt, B. Meyers, S. Harris and T. Huang: Automatic Face and Gesture Recognition (2000) 468. DOI:10.1109/AFGR.2000.840676

[4] G. P. Stein, M. Ofer and S. Amnon: Intelligent Vehicles Symposium (2003) 120. DOI:10.1109/IVS.2003.1212895

[5] L. Zhiying, C. Han and L. Lin: Computer Application and System Modeling (2012) 1060

[6] S. B. Gokturk, H. Yalcin and C. Bamji: IEEE Computer Vision and Pattern Recognition (2004). DOI:10.1109/CVPR.2004.17

[7] D. Muller, J. Pauli, C. Nunn, S. Gormer and S. Muller-Schneiders: Intelligent Transportation Systems (2009) 1. DOI:10.1109/ITSC.2009.5309851

\section{Introduction}


vehicle distance measurements may lead to severe accidents. The sensors for vehicle distance measurement are categorized into two classes [1]: (a) active depth detection system, e.g., radar, lidar and Time Of Flight (TOF) sensors (b) passive depth detection system, e.g., CCD camera. Active sensors can measure the distance very accurately but they are very expensive and the interference from similar systems is one of the major drawbacks. Passive sensors require more computation time and very sensitive to illumination condition. Stereo camera measures depth based on the disparity and it has some drawbacks; (1) the errors in distance measurements increase in polynomial manner proportional to the distance [2], (2) the accuracy of the disparity can be degraded under the low or high light conditions. Likewise, if the texture feature is too sparse or too dense, the disparity calculation problems are very difficult to solve [3]. Monocular distance measurement typically utilizes prior knowledge [4] and conducts camera calibration [5]. This approach identifies vehicles with already known knowledge such as edges, shapes, shadows. It assumes the position and the direction of camera is fixed and estimates the distance by using pixel distance and calibration parameters. However, when the position or direction of the camera is changed, recalibration is required once again. Due to the inherent prior knowledge, e.g., when shapes are occluded or illumination conditions are changed, the performance of the algorithm can be poor [6].

In this paper, we propose a new method for measuring the distance to the front vehicle based on monocular camera and a line laser. The benefits of the proposed method are as follows: (1) Proposed method is more accurate than disparity-based method. The errors in distance measurement was less than $4 \mathrm{~m}$ during the entire experiments. (2) It performs well even in night since both laser line and lane markings are visible in the night.

\section{Proposed distance measurement algorithm}

The scheme of the proposed method is as follows: a laser line is emitted in the near front of the vehicle on the road, and a monocular camera captures a sequence of images including the scene of the laser line and the road. We have three assumptions as follows: (1) lane detection system provides the information about the lane width. (2) the width of the laser line illuminated on the road is either measured directly or calculated by using the ratio between the width of the laser line and the lane width that is already known. It is reasonable since, depending on the road type, the width of lane markings follows the traffic regulations in general. The information about the lane width can be provided by geographic information system for transportation (GIS-T). (3) the lane width on the laser line is the same as the lane width on the rear-end point of leading vehicle. Fig. 1(a)-(b) depicts the device set-up and (c) explains the definition of the symbols and imaging geometry. The pinhole camera model gives [7]:

$$
\omega_{V}(t)=\frac{f \cdot W_{V}}{Z_{V}(t)}, \quad \omega_{L}(t)=\frac{f \cdot W_{L}}{Z_{L}(t)}
$$



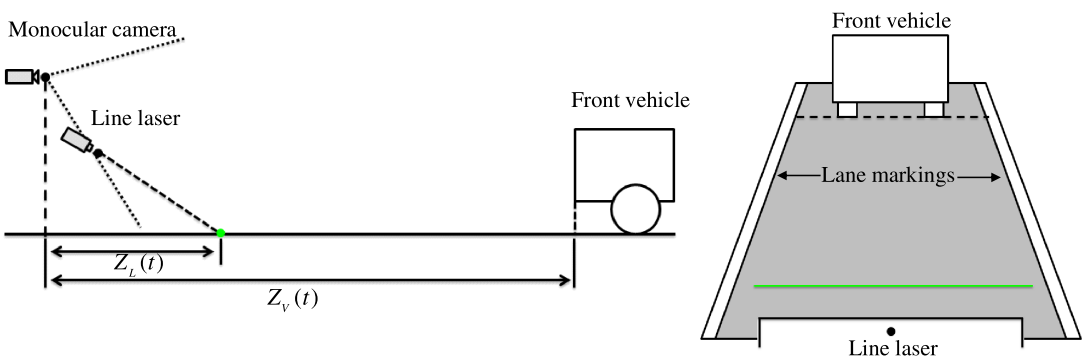

(a) Device setup (side view)

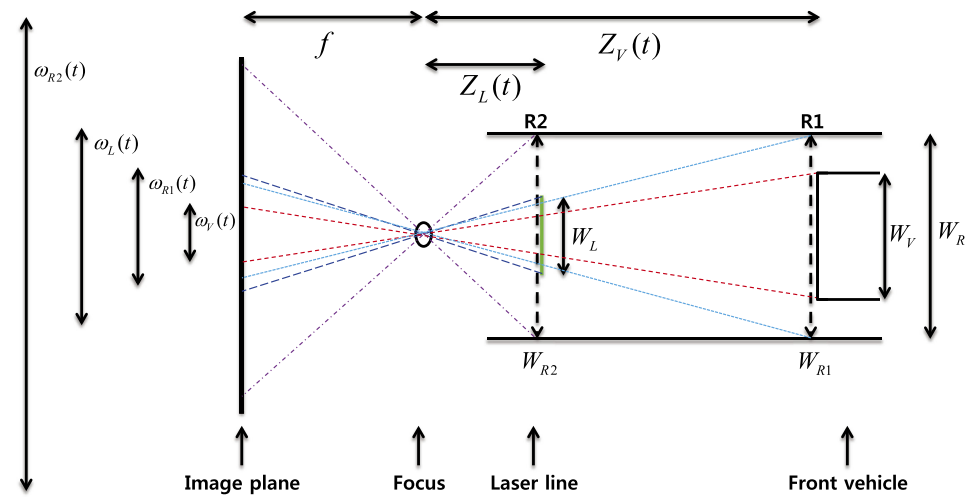

(c) The definition of symbols and imaging geometry

Fig. 1. Schematic diagram of the imaging geometry.

where, $W_{V}$ and $W_{L}$ mean the width of the vehicle and the lane, and $\omega_{V}(t)$ and $\omega_{L}(t)$ represent the width of the vehicle and the lane in the image plane, respectively. $Z_{V}(t)$ and $Z_{L}(t)$ are the distances from the camera to the front vehicle and to the line laser, and finally, $f$ means the focal length. According to the assumption $\mathrm{W}_{\mathrm{R} 1}=\mathrm{W}_{\mathrm{R} 2}=\mathrm{W}_{\mathrm{R}}$, we can induce the following equations:

$$
\frac{\omega_{R 2}(t)}{\omega_{L}(t)}=\frac{W_{R}}{W_{L}}, \quad \frac{\omega_{R 1}(t)}{\omega_{V}(t)}=\frac{W_{R}}{W_{V}}
$$

where, $\omega_{R 1}(t)$ and $\omega_{R 2}(t)$ are the lane width of the point R1 and R2 in the image plane. Following equations come from (2) and (4) is induced from (3):

$$
\begin{gathered}
W_{R}=\frac{W_{L} \cdot \omega_{R 2}(t)}{\omega_{L}(t)}, \quad W_{V}=\frac{W_{R} \cdot \omega_{V}(t)}{\omega_{R 1}(t)} \\
W_{V}=W_{L} \cdot \frac{\omega_{R 2}(t) \omega_{V}(t)}{\omega_{R 1}(t) \omega_{L}(t)}
\end{gathered}
$$

Rearranging (1) according to $Z_{V}(t)$ and substituting (4) in (1), we can get (5) comprised of focal length, width of the line laser, and three measurable variables from the image $\left(\omega_{R 1}(t), \omega_{R 2}(t)\right.$ and $\left.\omega_{L}(t)\right)$ as below:

$$
Z_{V}(t)=\frac{f \cdot W_{V}}{\omega_{V}(t)}=f \cdot W_{L} \frac{\omega_{R 2}(t)}{\omega_{R 1}(t) \omega_{L}(t)}
$$

To make units consistent in (5), dividing on the right-hand side of equation by pixel size $(p)$ gives $Z_{V}(t)$ in meters. Finally, for the enhancement of accuracy, by multiplying the calibration parameter $k$ we get (7).

$$
Z_{V}(t)=k \cdot f \cdot W_{L} \frac{\omega_{R 2}(t)}{\omega_{R 1}(t) \omega_{L}(t)} \cdot \frac{1}{p}
$$




$$
Z_{V}(t)=\frac{\omega_{R 2}(t) \cdot C}{\omega_{R 1}(t) \omega_{L}(t)}, \quad C=\frac{k f W_{L}}{p}
$$

Stereo camera-based distance measurement is shown as below and Fig. 2 depicts the principle:

$$
Z=B \cdot f / d
$$

where, $B$ represents the base line, the distance between two monocular cameras, and $d$ means the disparity, the difference between two images in the pixel unit. To get the value of $Z$ in meter unit, the right-hand side of equation should be divided by the pixel size.

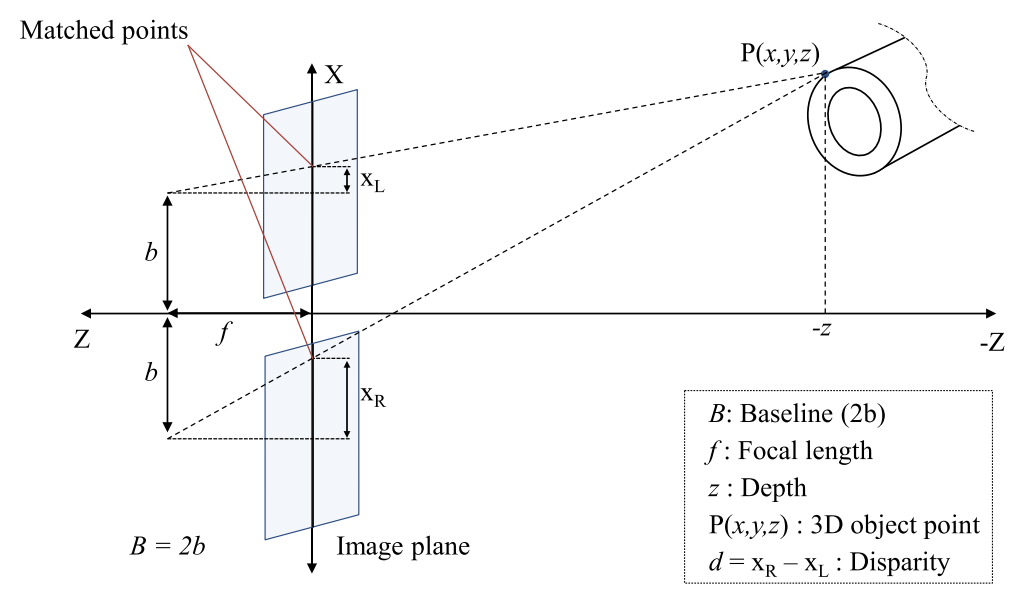

Fig. 2. Distance calculation based on the disparity map.

\section{Experiments and evaluation}

\subsection{Experimental system setup}

For the experiment, we installed a monocular camera and a line laser in the vehicle as well as a stereo camera to evaluate of the performance as shown in Fig. 3. We regarded the data from a lidar as ground truth, and made all the data synchronized. The specification of the sensors used in the experiments is shown in Table I.

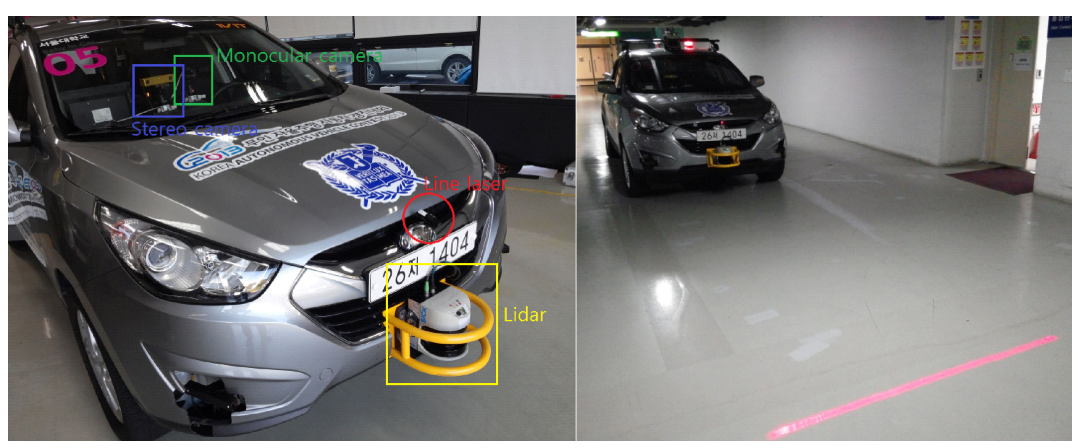

Fig. 3. Experimental system setup. 
Table I. Sensor specification

\begin{tabular}{|c|c|c|}
\hline Sensor & Model & Specification \\
\hline $\begin{array}{c}\text { Monocular } \\
\text { camera }\end{array}$ & $\begin{array}{c}\text { FL3-GE-03S2C-C (Camera) } \\
\text { DF6HA-1B (Lenz) }\end{array}$ & $\begin{array}{r}648 \times 488(82 \mathrm{fps}), 7.4 \mu \mathrm{m} \text { square pixel, } \\
\text { Focal length: } 6 \mathrm{~mm}\end{array}$ \\
\hline Line laser1 & BLSI65100A-L30 & $100 \mathrm{~mW}, 30^{\circ}$, red \\
\hline Line laser2 & SEI53100-L50 & $100 \mathrm{~mW}, 50^{\circ}$, green \\
\hline $\begin{array}{c}\text { Stereo } \\
\text { camera1 }\end{array}$ & BB2-08S2C-38 & $\begin{array}{c}1032 \times 776(20 \mathrm{fps}), 3.8 \mathrm{~mm}, 65 \text {-deg HFOV, } \\
4.65 \mu \mathrm{m} \text { square pixel, Baseline: } 12 \mathrm{~cm}\end{array}$ \\
\hline $\begin{array}{c}\text { Stereo } \\
\text { camera3 }\end{array}$ & BB2-08S2C-38 & $\begin{array}{c}640 \times 480(20 \mathrm{fps}) \\
\text { Same with stereo camera1 }\end{array}$ \\
\hline $\begin{array}{c}\text { Stereo } \\
\text { camera2 }\end{array}$ & VSTC-P250 & $\begin{array}{c}640 \times 480(20 \mathrm{fps}), 8 \mathrm{~mm}, \\
6 \mu \mathrm{m} \text { square pixel, Baseline: } 26 \mathrm{~cm}\end{array}$ \\
\hline Lidar & LMS511 outdoor & $\begin{array}{c}\text { Measurement range: } \text { up to } 65 \mathrm{~m}, \\
\text { Field of view: } 190^{\circ}\end{array}$ \\
\hline
\end{tabular}

\subsection{Experimental results}

We collected two sets of experimental data for daytime and nighttime in the highway. For nighttime dataset, we used the stereo camera1 and line laser1 while stereo camera 2 and line laser 2 were used for daytime dataset. Fig. 4 is the snapshot of each datasets. From left to right, monocular camera with line laser image, stereo disparity image, the distance image generated by using the data from lidar. We adopted the built-in disparity map provided by the camera.

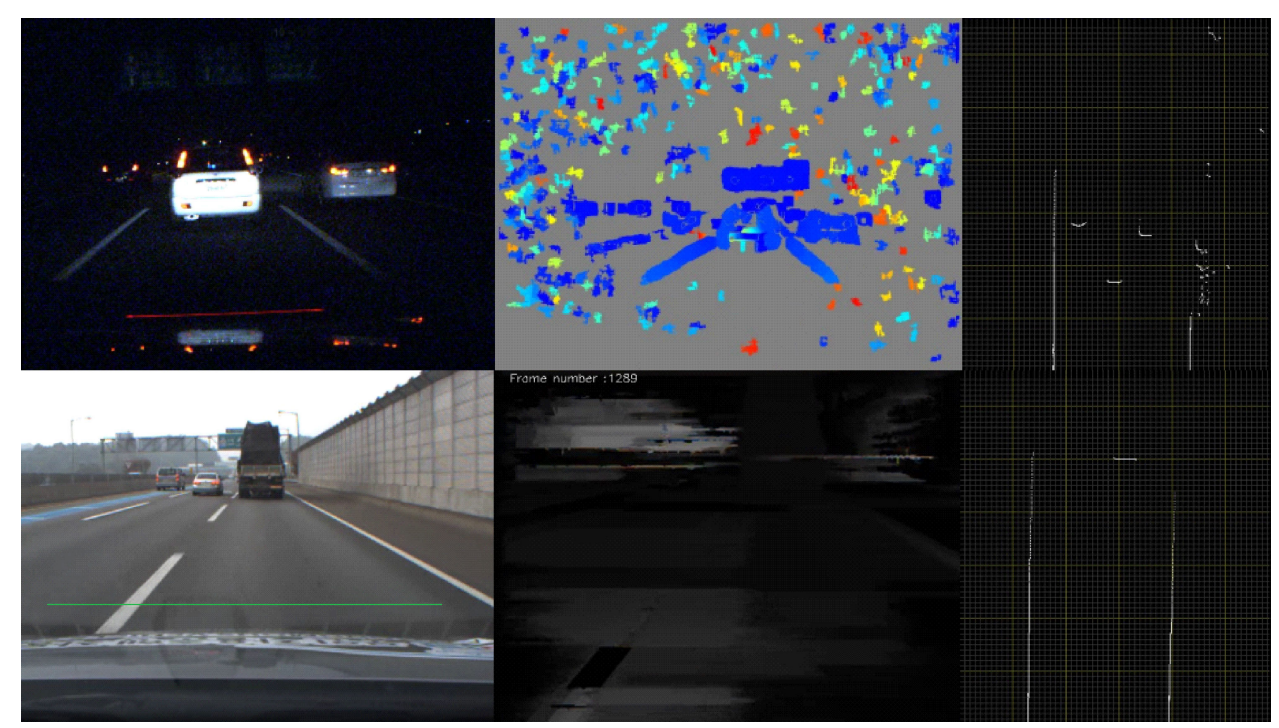

Fig. 4. The snapshot of the dataset.

According to the experimental results as shown in Fig. 5, the proposed method provides higher accuracy as compared to the stereo camera-based method. Fig. 5(a) depicts the sequence of distance measurements in daytime highway while Fig. 5(b) depicts the nighttime highway situation. The distance was not constantly decreased or increased during the experiments. 


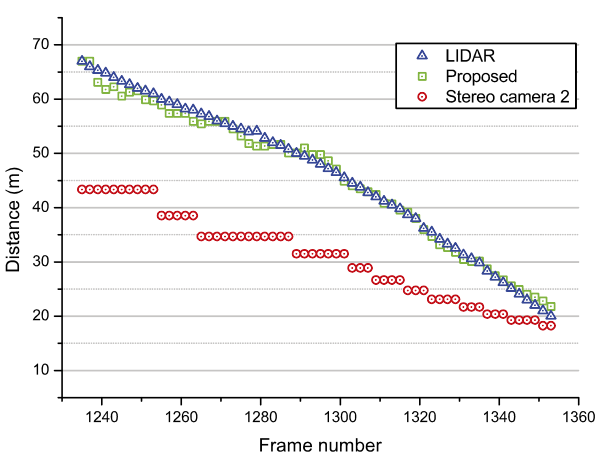

(a) Daytime distance measurement

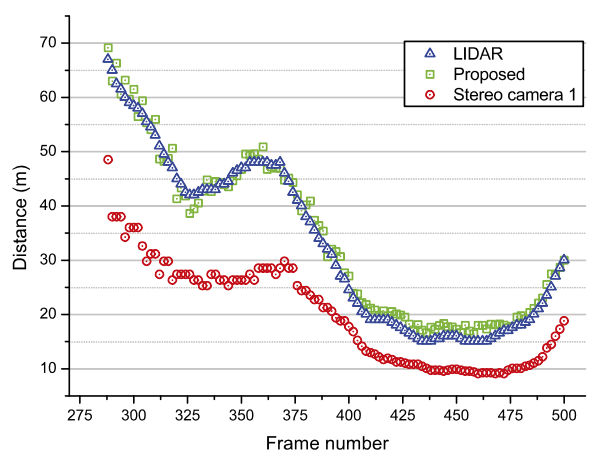

(b) Nighttime distance measurement

Fig. 5. Distance measurement results.

Average distance measurement error of experiments is shown in Table II. The results show that the proposed method is more accurate than stereo camera and the distance measurement error is less about ten times on average. The results in Table II show that the average error in experiment1 $(1.05 \mathrm{~m})$ is lower than in experiment2 $(1.79 \mathrm{~m})$, though the illumination conditions in experiment1 is better than in experiment2. This is due to the fact that the resolution of camera1 used in experiment1 is 1.6 times lesser than camera2 used in experiment2. Since the distance is inversely proportional to the disparity and the number of pixel is limited, lower resolution has more distance error.

Table II. Average error in distance measurement

\begin{tabular}{|c|c|c|}
\hline & Proposed & Stereo camera \\
\hline Experiment1 (daytime) & $1.05 \mathrm{~m}$ & $15.11 \mathrm{~m}$ \\
\hline Experiment2 (nighttime) & $1.79 \mathrm{~m}$ & $12.74 \mathrm{~m}$ \\
\hline Average & $1.42 \mathrm{~m}$ & $13.92 \mathrm{~m}$ \\
\hline
\end{tabular}

The error in distance measurement-to-distance relationship is shown in Fig. 6. For comparison study, we adopted the theoretical distance measurement error from the datasheets provided by the manufacturer. Stereo camera3 has the same specification as stereo camera1 except the resolution. But the resolution of stereo camera1 is same as monocular camera (Refer Table I). Comparison results show that the distance measurement error of the proposed method is lower than the other methods. In case of lidar, the error is $\pm 2.5 \mathrm{~cm}$ in $1-10 \mathrm{~m}$ range, $\pm 3.5 \mathrm{~cm}$ in $10-20 \mathrm{~m}$ range and $\pm 5 \mathrm{~cm}$ in $20-30 \mathrm{~m}$ range.

\section{Conclusion}

In this paper, we proposed a new method for the longitudinal inter-vehicle distance measurement by using monocular camera and line laser. Experimental results show that the proposed method exhibits high accuracy as compared to the stereo camera-based method. The benefits of the proposed method are in summary: (1) the proposed method still shows high accuracy in nighttime 


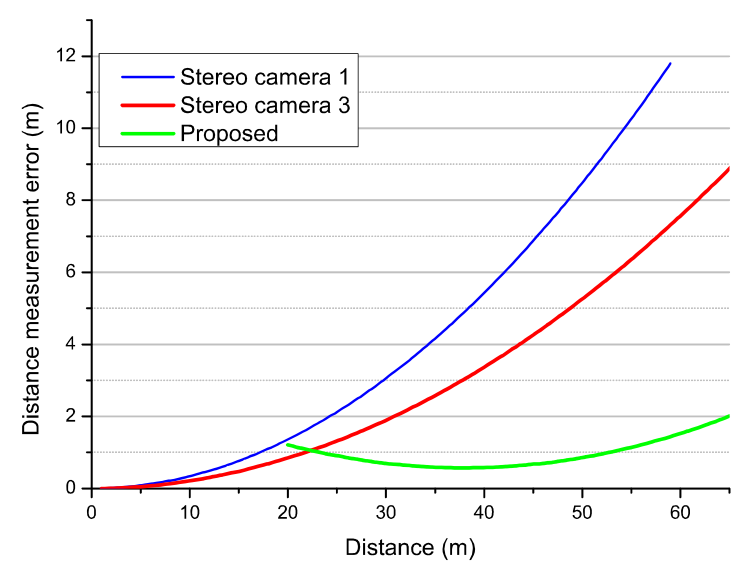

Fig. 6. The comparison results of the error in distance measurement as compared to the lidar for the distance variations.

as compared to the stereo camera-based method which suffer from low illumination conditions. Since, the proposed method utilized a line laser and lane markings that are visible well in night. (2) The distance measurement error of the proposed method is lesser in several meters while the stereo camera-based method increases with distance proportionally to the square of the distance.

\section{Acknowledgments}

This research was supported by Basic Science Research Program through the National Research Foundation of Korea (NRF) funded by the Ministry of Education, Science and Technology (2012-0000918), and partially by Institute of New Media and Communications, Seoul National University. 